\title{
Die akute Osteitis
}

\section{Volkmar Heppert, Andreas Wentzensen}

Trotz verbesserter atraumatischer, sogenannter minimalinvasiver Operationsmethoden, trotz verbesserter Implantate und adäquater Antibiotikaprophylaxe treten weiterhin postoperative Infektionen auf. Jede postoperative Wundinfektion bedeutet für den Patienten eine schwere Komplikation, die sein/ihr weiteres Leben entscheidend beeinflussen kann. Für den Patienten, den diese Komplikation persönlich betrifft, ist es unverständlich, wie es dazu hat kommen können. Neben der persönlichen Verunsicherung spielen sozialmedizinische Aspekte eine wesentliche Rolle in der Gesamtproblematik. Funktionelle Folgeschäden, persistierende Schmerzen und längere stationäre Behandlungszeiten sind die Folge. In seltenen Einzelfällen, wie zum Beispiel beim Gasbrand oder der nekrotisierenden Fasciitis, kann sogar der Tod des Patienten resultieren. Folgen zu spät erkannter Infektionen führen daher oft zu erheblichen Haftpflichtforderungen [1]. Die Infektion nach Osteosynthese ist ein multifaktorielles Geschehen und stellt nach wie vor große Anforderungen an den behandelnden Arzt. Die einwirkende

\section{Zusammenfassung}

\section{Epidemiologie}

Infektionen nach Osteosynthese sind relativ seltene Komplikationen. Bei so genannten Elektiveingriffen werden in der Literatur Infektraten von $0,1-1,7 \%$ beschrieben. Für geschlossene Frakturen gelten $1-5 \%$ als realistisch. Beim Extremitätentrauma gelten jedoch ganz andere Ausgangsbedingungen. In Ausprägung

OP-JOURNAL 2005; 21: 224-230

(c) Georg Thieme Verlag KG Stuttgart · New York entzündliche Noxe führt erst zeitlich verzögert zu klinisch apparenten Symptomen. Da Infektionen nach Osteosynthese, dank oben genannter Fortschritte der modernen Chirurgie, relativ seltene Komplikationen geworden sind, werden die Frühsymptome nach wie vor oft nicht rechtzeitig korrekt interpretiert. Die Frühdiagnostik einer solchen Infektion ist zusammen mit einem daraus resultierenden aggressiven chirurgischen Vorgehen das entscheidende Kriterium für das spätere Endergebnis des Patienten. Für die Versicherungsträger erzeugt ein Infektverlauf eine erhebliche finanzielle Mehrbelastung. Zunehmend sind Tendenzen nachweisbar, diese Mehrbelastung auf den vermeintlichen „Verursacher“, die Klinik, abzuwälzen. Das DRG-System unterstützt dies dahingehend, dass der Infekt, der innerhalb der „Oberen Grenzverweildauer" (OGD) auftritt und zur operativen Revision zwingt, nicht zusätzlich vergütet wird. Die Mehrkosten bedeuten damit für die erstbehandelnde Klinik eine erhebliche finanzielle Belastung. Dies muss dazu führen, dass der Infektvermeidung eine wesentliche Schlüsselstellung zu kommt. hier noch neue zeitliche Definitionen in der Zukunft entstehen, die die akute Osteitis wirklich nur auf die direkte postoperative Wundinfektion limitiert.

\section{Pathophysiologie}

Die alleinige Anwesenheit eines Bakteriums erzeugt noch keine Infektion. Ca. $70 \%$ der offenen Frakturen sind bekanntermaßen kontaminiert, aber die Infektrate ist im klinischen Alltag deutlich geringer [8].

Nach wie vor sind die zugrunde liegenden Mechanismen der Enstehung einer Infektion ungeklärt. Immer mehr Arbeiten beschäftigen sich mit Substanzen aus der Molekularbiologie wie Cytokinen und Wachstumsfaktoren. Der Vorgang ist aber so komplex, dass wir weit davon entfernt sind, die Zusammenhänge wirklich zu verstehen.

Nach wie vor sind verschiedene kausale Faktoren nicht wegzudiskutieren $[7,8]$

- Erreger

- Zahl und Virulenz der Keime

- Patientenfaktoren

- Lokale Wundsituation

Die Affinität mancher Erreger zu Rezeptoren von Wirtsproteinen wie Kollagen und insbesondere Fibronectin, welche bereits in der Frühphase nach Trauma in der Wunde zu finden sind, führt zur Keimansammlung und Kolonisierung im Wundgebiet. Lokale Durchblutungsstörungen infolge Trauma führen zusätzlich zur Gewebsnekrose und zu Sequestern, die dann im Sinne einer Fremdkörperwirkung - insbesondere bei einliegendem Osteosynthesematerial - wirken.

Nicher definiert ist die Frage, unter einer akuten Osteitis zu verstehen ist. Manche Autoren sehen eine Frist begrenzt auf einen Zeitraum von 8 Wochen nach dem Trauma [6], aber diese Zeit ist willkürlich festgesetzt. Modernere Arbeiten aus der Endoprothetik lassen erkennen, dass der initiale Zeitraum von 3 Monaten sich mittlerweile auf 4 Wochen verkürzt hat. Es ist davon auszugehen, dass
Gerade ein einliegendes Implantat bedeutet hierbei einen wesentlichen pathogenetischen Faktor. Darüber hinausgehend konnte eindrucksvoll nachgewiesen werden, dass Bakterien, die adhärent auf Oberflächen von Implantaten haften, eine Resistenz gegen die bakterizide Wir- 


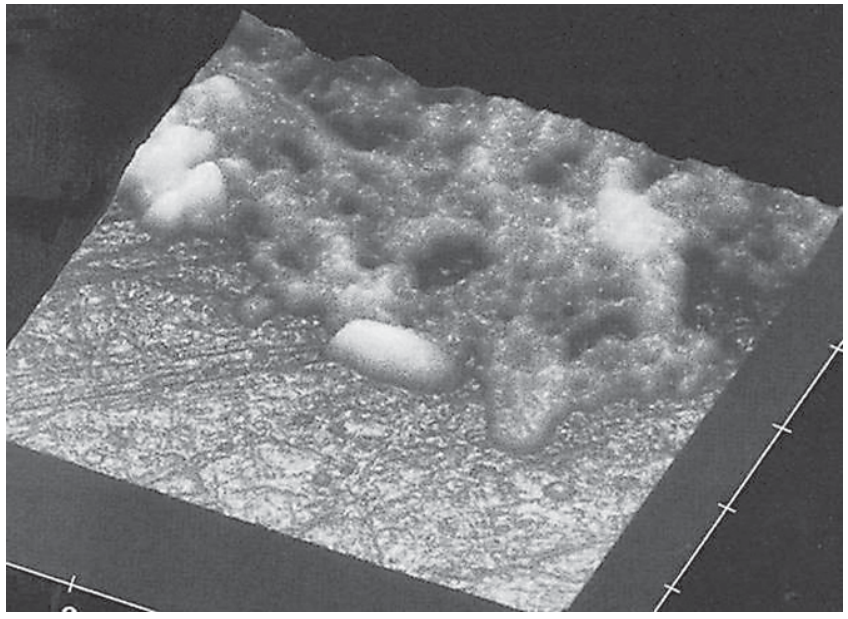

Abb.1 Biofilm Elektronenmikroskop auf Metallimplantat.

kung von Antibiotika entwickeln können und dieses veränderte Resistenzmuster sogar beibehalten, wenn sie sich von der Oberfläche des Implantates entfernt haben [9]. Liegt die Verdoppelungszeit von Bakterien üblicherweise bei ca. $35 \mathrm{~min}$, so reduziert sie sich bei liegendem Implantat auf bis zu 4 Stunden, je nach Erreger.

Die Fähigkeit vieler Erreger, einen so genannten Biofilm zu produzieren, schützt diese dann zusätzlich vor dem Angriff von Antibiotika [10,11]. Dieser Biofilm besteht vorwiegend aus extrazellulären Zuckern. Ein stabiles mikrobiologisches System kann unterhalb dieses Biofilms entstehen und dort jahrelang klinisch unauffällig bleiben. $\mathrm{Zu}$ einem späteren Zeitpunkt - aus bisher ungeklärter Ursache - können die Bakterien dann jederzeit wieder aktiv werden [11]. Und dies muss Eingang finden in unsere therapeutischen Überlegungen, da dieser Biofilm wesentlich verantwortlich ist für die Problematik des Infektes. bei einliegendem Implantat (Abb.1).

Der Biofilm auf dem Implantat ist das wesentliche Problem beim Infekt nach Osteosynthese.

\section{Keimspektrum}

Staphylokokken zeigen eine ganz besondere Affinität zu Implantaten, egal ob sie aus Metall oder Kunststoff sind. Sie sind bei postoperativen Infektionen die am häufigsten anzutreffenden Erreger. In den letzten Jahren ist im speziellen Krankengut unserer Sektion Posttraumatische Osteitis eine Zunahme von Enterokokken sowie Streptokokken zu verzeichnen. Der MRSA/ORSA zeigt jährlich einen höheren Anteil bei den Erstisolaten von Staphylokokken auf $>30 \%$ in Deutsch- land. Er spielt aber beim Frühinfekt mit $<5 \%$ eine untergeordnete Rolle.

\section{Diagnostik}

Klinische Zeichen der beginnenden Infektion sind oft nur diskret ausgeprägt. Deshalb muss man, wie Bühler es formuliert, „Erkennen, Bekennen, Handeln“ [12]. Der Schlüssel zum Erfolg liegt darin, die Komplikation als solche, so früh es geht, erkennen zu wollen. Der hochseptische Verlauf ist dabei problemlos, aber gerade die unterschwelligen Ausprägungsformen gilt es zu entdecken. Der Operateur muss dabei aber auch bereit sein, sich eine Komplikation einzugestehen.

Ein an der Operation nicht beteiligter Arzt sollte die Indikation zur Revision stellen. Dies sollte auch dann gelten, wenn der Chef selbst operiert hat.

\section{Diagnostik}

\section{Labor}

Unter Berücksichtigung von Sensitivität, Spezifität, Verfügbarkeit und insbesonde-

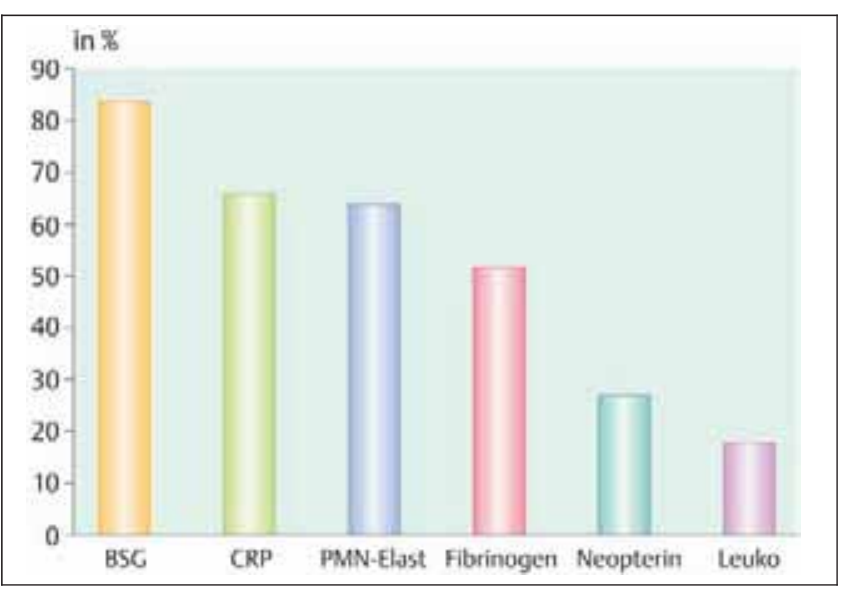

re Kostengesichtspunkten stellt das CRP für die klinische Praxis den geeignetsten Marker dar [6]. Einzelheiten bezüglich der diagnostischen Marker siehe Abb. 2.

\section{Bildgebende Diagnostik}

- Sonographie: Weichteilinfekte oder Abszesse lassen sich sensitiv nachweisen. Ansonsten hat diese Untersuchung keinen Stellenwert.

- Magnetresonanztomographie (MRT): Die MRT spielt bei der akuten Osteitis keine wesentliche Rolle, sie ist das Diagnoseinstrument bei der chronischen Osteitis. In den ersten 6 Wochen nach einem operativen Eingriff sind nämlich kaum verwertbare Befunde zu erzielen, da sich die OP-Folgen nicht von entzündlichen Veränderungen trennen lassen. Einliegende Implantate können je nach Metall bzw. Liegedauer zudem die Untersuchung unmöglich werden lassen, beziehungsweise Artefakte erzeugen, die eine Interpretation nicht mehr zulassen.

- Szintigraphie: Sie besitzt in der Diagnostik der Frühinfektion keinen Stellenwert. Auch hier wird in der Frühphase nach OP immer eine Anreicherung erfolgen, auch wenn kein Infekt vorliegt.

\section{Therapie}

Alle therapeutischen Bestrebungen müssen auf eine Eradikation der Infektion gerichtet sein. Der frühestmögliche Zeitpunkt der Revision ist der Beste.

Nur ein erfahrener Operateur sollte diese Revisionseingriffe vornehmen.

Ziel der Operation muss es sein, alles infizierte nekrotische Gewebe zu entfernen und das gelingt eigentlich nie. der Entzündungsmarker in \% (5) 


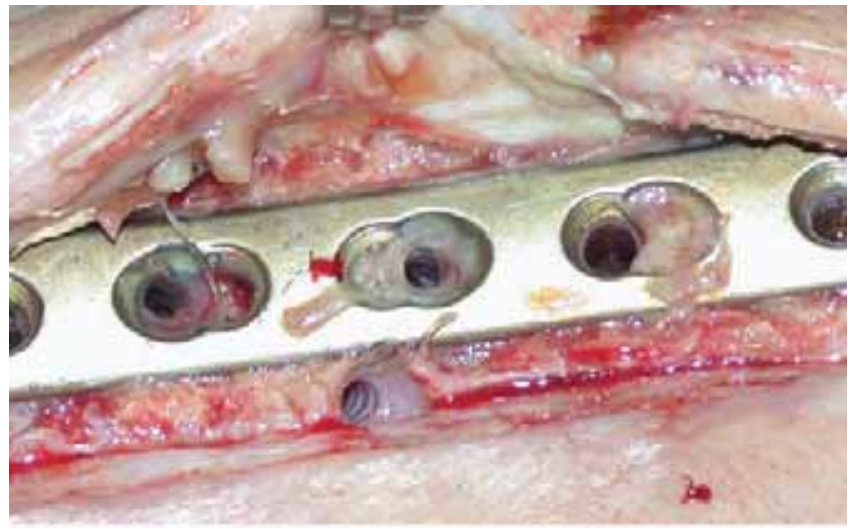

Abb. 3a Akuter Plattenlagerinfekt an der Tibia.

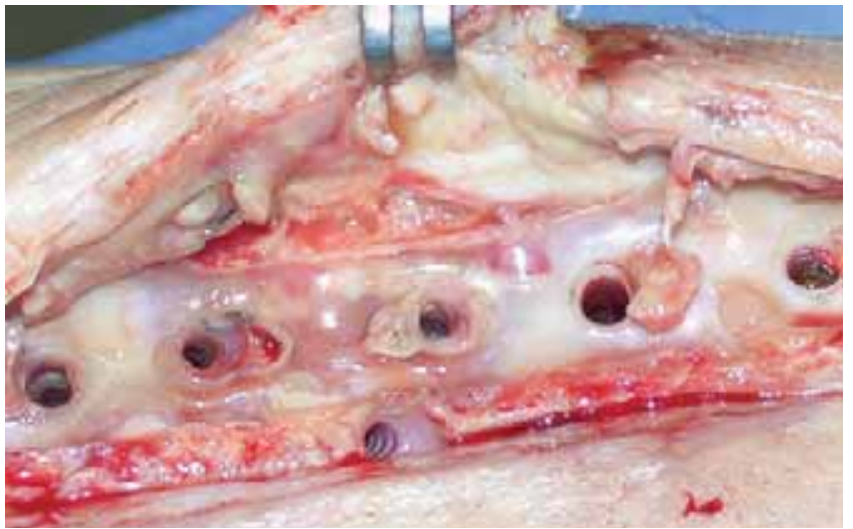

Abb. 3b Nach Metallentfernung zeigt sich einerseits unter der Platte ein Infektrasen, andererseits kann man erst jetzt die Schraubenkanäle ins Debridement einbeziehen.
Mit folgenden Problemen muss sich der Operateur auseinander setzen:

- Lokalisation der Infektion

- Weichteilbedeckung

- Implantat

In diesem Spannungsfeld gibt es verschiedene Therapieoptionen:

\section{Debridement}

Das Debridement muss so radikal und umfassend, wie irgend machbar erfolgen. Es ist der Schlüssel zum möglichen Erfolg. Aber hier entstehen mehr Fragen als Antworten:

- Wie kann der Operateur unter der Platte debridieren, wo sich der Infekt genauso manifestiert hat, wie auf der Platte? (Abb. 3a u.b)

- Wie kann dies bei einliegendem Marknagel oder Endoprothese erfolgen, wenn man den Nagel bzw. die Prothese nicht wechselt? (Abb.4)

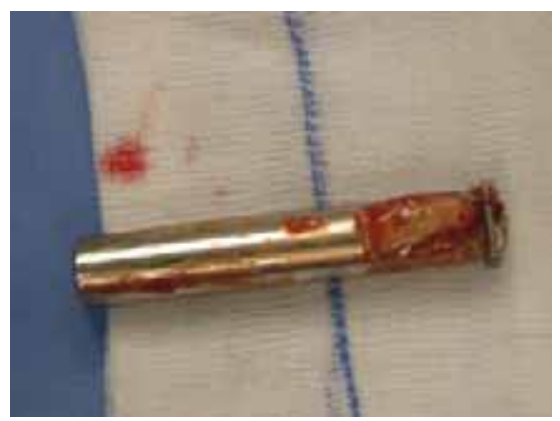

Abb.4 Verriegelungsbolzen einer gekoppelten Knietotalendoprothese. Dies kann beim Debridement mit Versuch des Erhaltes des Implantates nicht erreicht werden. Deshalb muss man immer die geeigneten Instrumente vorhalten, bevor man eine Revision beginnt.
Arens et al. [13] konnten nachweisen, dass ein einmal bakteriell besiedeltes Implantat nicht mehr keimfrei in situ werden kann. Costerton [10] konnte zeigen, dass eine Einwirkzeit eines Desinfektionsmittels auf ein infiziertes Implantat nur zu einem Absterben der oberen Bakterienschichten führt. Die in der Tiefe lebenden überstehen dies mühelos. Welcher Operateur aber hat bei der Revision jemals 90 min desinfiziert und hat dann doch keine Garantie auf Erfolg?

\section{Vakuumversiegelung (VVS)}

Die VVS ist unseres Erachtens nach eine zunehmend überschätzte Methode. Sie ist unschlagbar im primären temporären hermetischen Verschluss einer Wunde. In unserem septischen Zentrum sehen wir aber zunehmend Patienten, bei denen die Anwendung und die Indikation zu kritisieren sind.
Manchmal findet sich gestörter Sekretabtransport (Abb.5). Die sich dann unter der Folie ansammelnde Flüssigkeit ist bester Nährboden für Erreger.

- Die Versiegelung erfolgt auf nicht radikal debridiertem Knochen (Abb.6a u.b).

- Die Versiegelung erfolgt oft mit versenktem Schwamm bei einliegendem Antibiotikumträger. Dies ist nicht nachvollziehbar, da das Antibiotikum kontinuierlich abgesaugt wird. Hier ist eine Kosten-Nutzenanalyse empfehlenswert (Abb. 7a u.b).

- Durch wiederholte VVS verliert der Patient wertvolle Zeit bis zur Sanierung (Abb.8a-c). Leider sind Fälle, wie der hier gezeigte keine Einzelfälle mehr.

VVS ersetzt nicht das radikale Debridement. Kombination von VVS mit lokalen Antibiotikumträgern ist unsinnig.

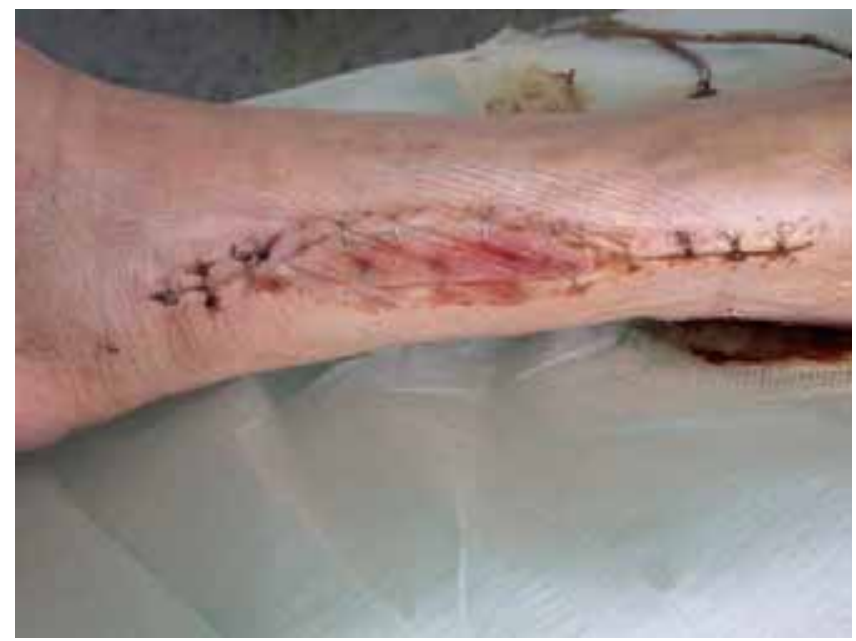

Abb. 5 Fehlender Sog führt zur Sekretbildung unter der Folie. 


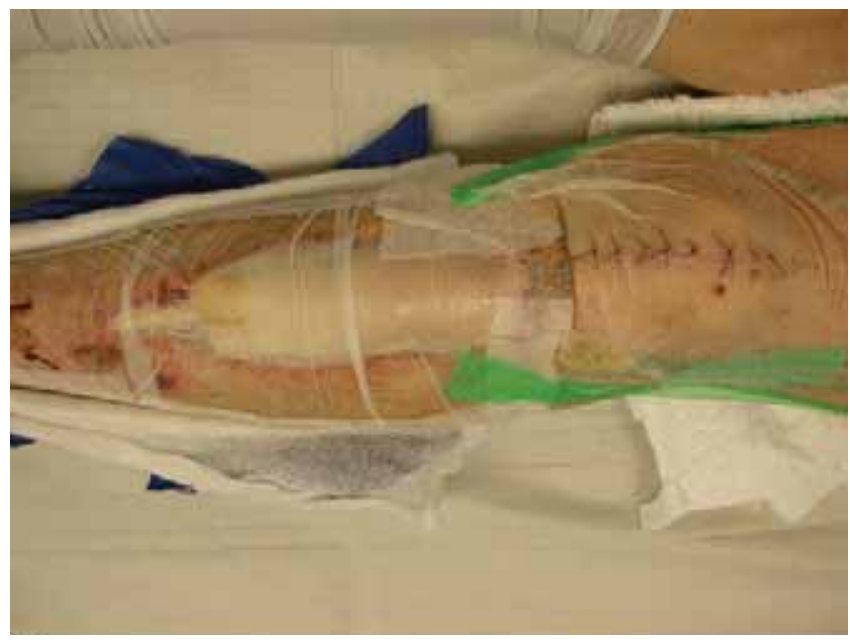

Abb.6a Großflächige Versiegelung bei Infekt/Defektsituation.

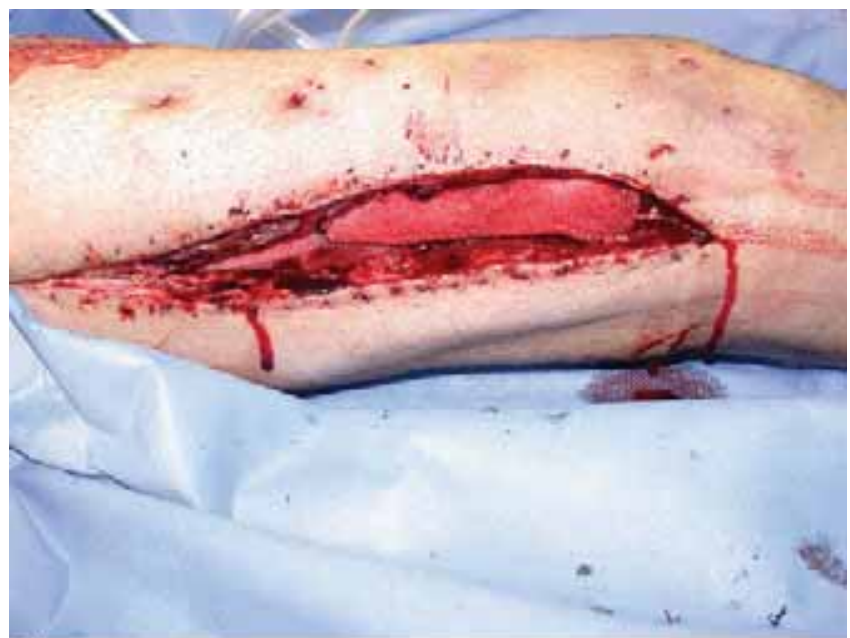

Abb. 7a Unter der verschlossenen Haut eingebrachte VVS.

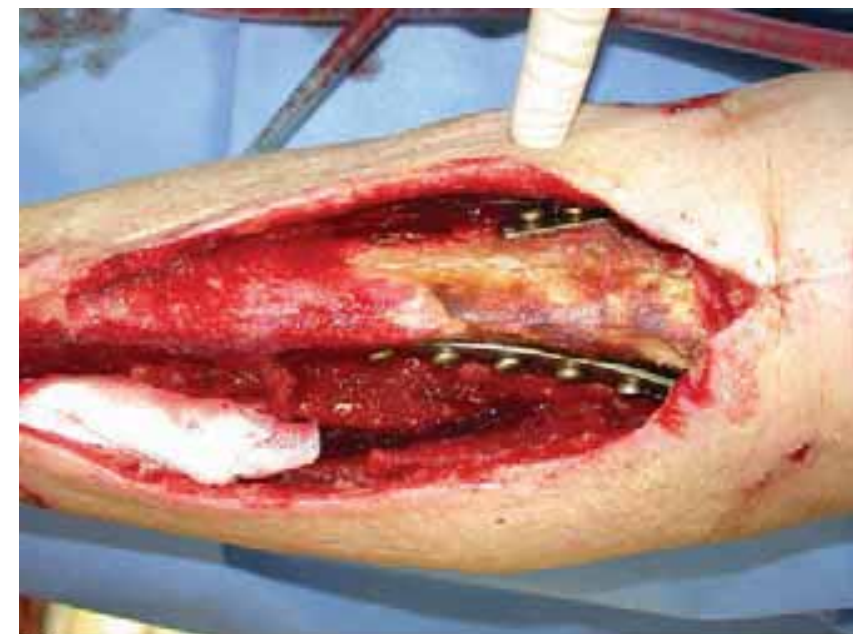

Abb.6b Die Nekrose des denudierten Knochens wurde belassen. Debridement vor Versiegelung!

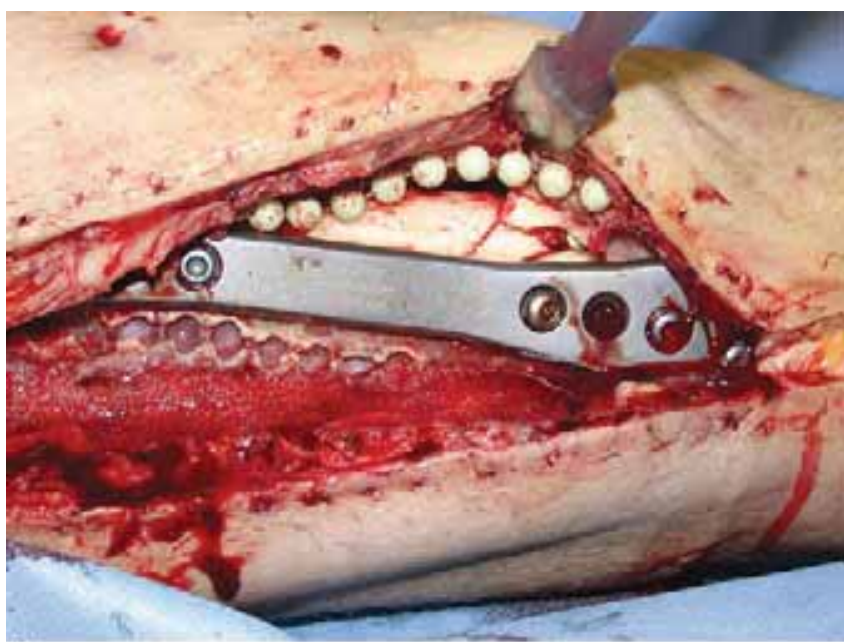

Abb. 7b Nekrotischer Knochen wurde belassen. Kombination von PMMA-Ketten mit VVS nicht sinnvoll.

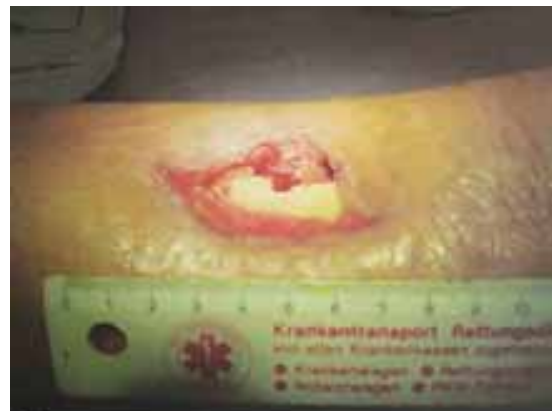

Abb. 8a Dokumentation einer Vakuumversiegelung. Beginn 11.4.2004 nach offener Unterschenkelfraktur. Einliegender UTN.

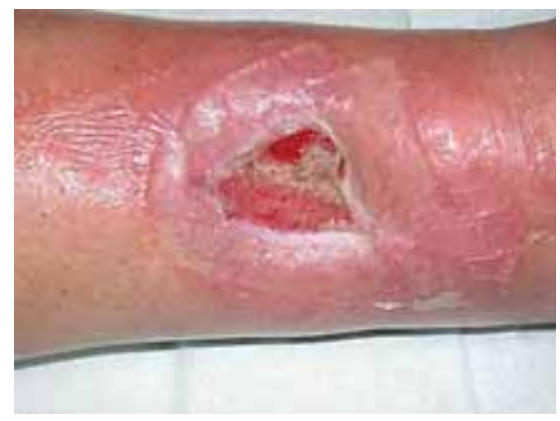

Abb. 8b Letzte VVS am 21.12.2004. Nach 8monatiger VVS hat die Patientin sich in unserer Klinik selbst vorgestellt.

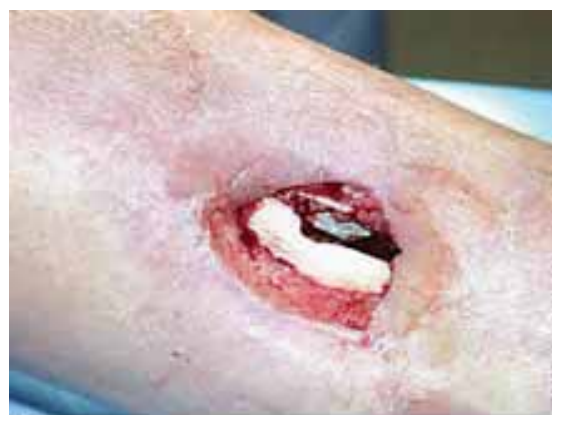

Abb. 8c Im OP zeigt sich die Osteonekrose des Knochens mit einliegendem Implantat. Staph. Aureus bei chronischem Markrauminfekt. Zeitverlust 8 Monate. 

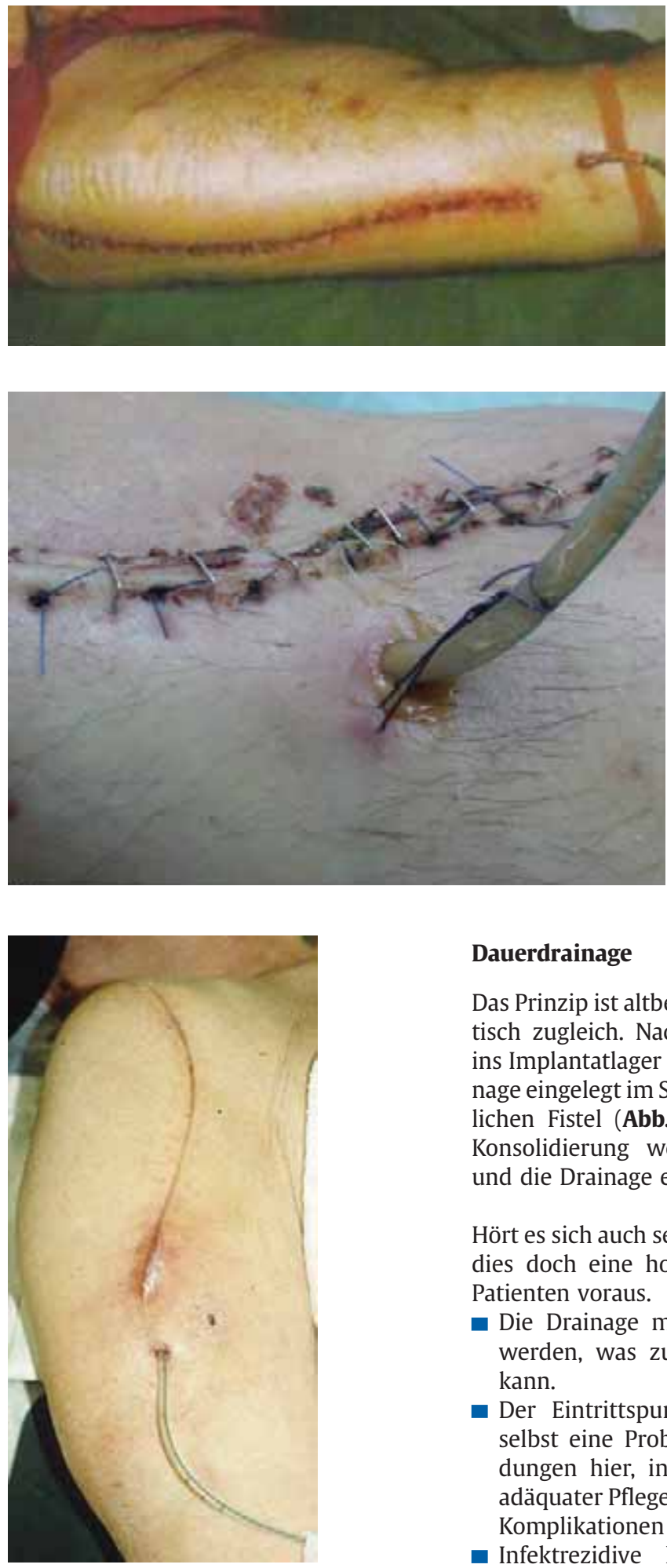

Abb.9c Einliegende Dauerdrainage mit Subkutanabszess am tiefsten Punkt der Wunde. Infektrezidiv.
Abb.9b Infekt an der Austrittsstelle der Drainage bei

Infektpersistenz.

Abb. 9a Reizfrei einliegende Dauerdrainage bei Femurfraktur.

\section{Dauerdrainage}

Das Prinzip ist altbewährt und problematisch zugleich. Nach Debridement wird ins Implantatlager eine dicke Redondrainage eingelegt im Sinne einer kontinuierlichen Fistel (Abb.9a). Mit knöcherner Konsolidierung werden das Implantat und die Drainage entfernt.

Hört es sich auch sehr einfach an, so setzt dies doch eine hohe Compliance beim Patienten voraus.

- Die Drainage muss täglich gelockert werden, was zur Dislokation führen kann.

- Der Eintrittspunkt der Drainage ist selbst eine Problemzone, da Entzündungen hier, insbesondere bei nicht adäquater Pflege, häufig anzutreffende Komplikationen sind (Abb.8b).

- Infektrezidive können auch unter Dauerdrainage eintreten (Abb.8c).

\section{Etappenlavage}

Bei diesem Konzept handelt es sich um programmierte Etappenlavagen in 2-3tägigen Abständen [14]. Bei jeder Revision wird ein Abstrich entnommen. Revisionen werden sooft wiederholt, bis 3 negative Abstriche vorliegen. Ist dies nach 4 Operationen nicht der Fall, wird das Implantat entfernt [4]. Nur ca. 30\% aller Implantate ließen sich in situ halten, aber eine chronische Osteitis wurde nicht beobachtet [14]. Dennoch hat auch dieses Konzept seine Kritikpunkte:

- Unter DRG-Gesichtspunkten rechnet sich dieses Vorgehen für die Klinik nicht mehr.

- Wenn man 3 negative Abstriche in Folge benötigt, würde dies rein rechnerisch bedeuten, dass bereits der 2 . Abstrich steril sein muss. Ist er es nicht und gebe ich mir maximal 4 Operationen [4] bevor das Metall entfernt wird, dann braucht man OP 3 und 4 nicht mehr durchführen, da das Implantat ohnehin entfernt wird.

- Der Patient verliert Zeit und empfindet jede erneute Revision als „wieder eine Operation". Wird dann das Metall doch entfernt, ist das Vertrauen in Methode und Arzt limitiert.

- Trotz manifester klinischer Infektion findet sich in $10-15 \%$ ein negativer Abstrich. Hier ist das Konzept klar zum Scheitern verurteilt.

Die Autoren dieses Konzepts berichten auf Kongressen inzwischen von deutlich schlechteren Ergebnissen mit der Etappenlavage. Nur noch ca. $15 \%$ der Implantate könnten so erhalten werden. Eine Erklärung für diese Verschlechterung der Ergebnisse - 35 versus 15\% - konnte bisher nicht geliefert werden.

Frühsymptome einer Infektion sind:

- Leukozytenzahl (unspezifisch)

- CRP (unter klinischen und Kostengesichtspunkten der z.Zt. günstigste Marker)

- Schmerz $\uparrow$, Schwellung $\uparrow$, Rötung $\uparrow$ Temperaturanstieg

Abstrich:

Grundsätzlich und unabhängig vom angewendeten Verfahren sind Abstriche aus den Weichteilen und dem Implantatlager zu entnehmen und möglichst mit Gewebeproben der Bakteriologie zuzusenden. Der übliche alleinige Abstrich aus scheinbar infizierter Flüssigkeit bringt in ca. 10-15\% keinen Keimnachweis. Der positive Befund ist DRG relevant.

\section{Implantat - belassen oder entfernen?}

Ein ungelöstes Problem findet sich nach wie vor, wenn ein Infekt auftritt und ein Implantat einliegt. Die grundlegenden Arbeiten der Bonner Arbeitsgruppe 


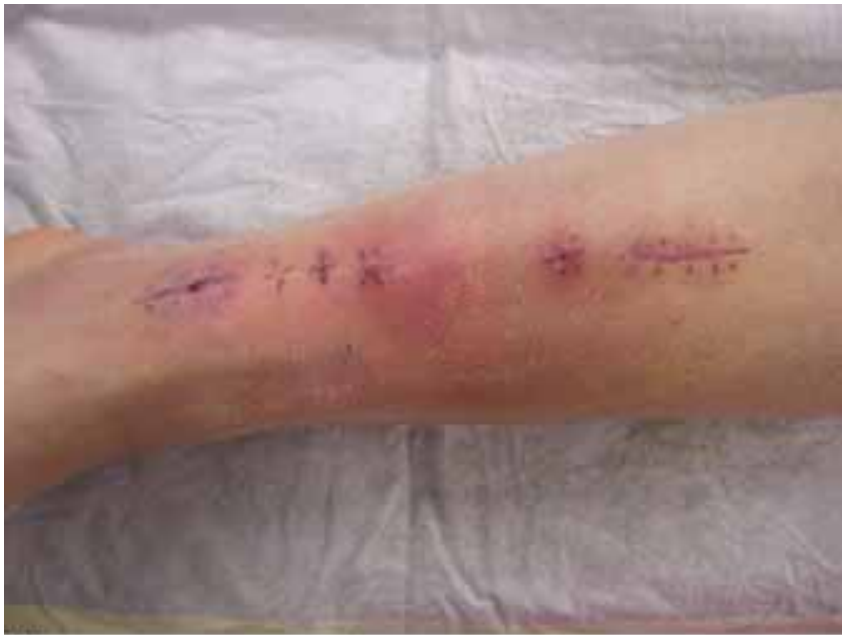

Abb.10a Frühinfekt nach eingeschobener Plattenosteosynthese an der Tibia.

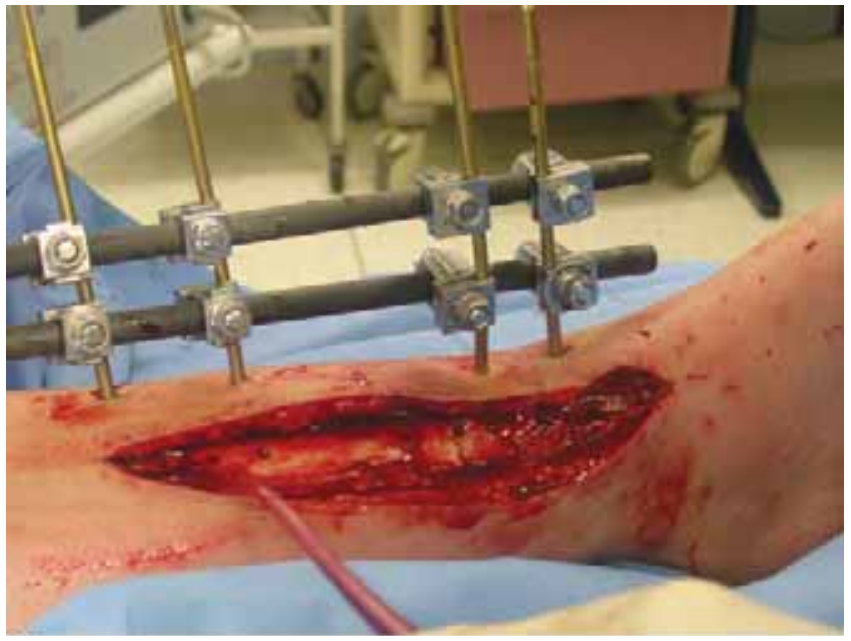

Abb.10b Metallentfernung bei gut durchblutetem Knochen. Umstieg ohne Repositionsverlust auf Fixateur externe. Problemloser weiterer Verlauf.
[13] demonstrierten nachdrücklich, dass ein einmal bakteriell besiedeltes Metallimplantat unabhängig von der Legierung nicht mehr steril wird. Bedenkt man die oben diskutierten pathophysiologischen Überlegungen, insbesondere unter dem Gesichtspunkt des „Radikalen Debridements" (Abb.3) und des Biofilms (Abb.1), so ist die sicherste Option die Metallentfernung im Infekt. Ob man dann nach wirklichem Debridement, ggfs. mit Aufbohren der Markhöhle etc. gleich oder sekundär die Reostensynthese durchführt, ist abhängig von der Fraktur, der Intensität des Infektes und der Erfahrung des Operateurs mit dieser Komplikation (Abb.10a u.b).

Auch hier gibt es Diskussionspunkte:

- Implantatwechsel ist ein für die Klinik kostenintensives Verfahren, welches sich unter DRG-Gesichtspunkten nicht rechnet. Das neue Implantat wird ja nicht separat vergütet.

- Bei den gelenkbildenden Frakturen, insbesondere nach zusätzlicher Spongiosaplastik, bedeutet die Metallentfernung oft einen Zusammenbruch der Reposition. Hier muss man im Einzelfall abwägen, allerdings zeigen die klinischen Resultate unseres Patientengutes, dass bei diesen Frakturen ein hohes Risiko der chronischen Osteitis besteht, wenn man das Implantat belässt.

- Das so genannte „Blitzen“ ist als Methode der Sterilisation in Deutschland nicht mehr erlaubt. Die validierte Aufbereitung eines entnommenen Implantats rechnet sich nicht, da für den Vorgang so viel Zeit benötigt wird, dass dies teurer ist, als ein neues zu verwenden.

\section{Antibiose}

Diese ist immer nur als Adjuvans zur chirurgischen Therapie zu sehen. Sowohl in der systemischen als auch lokalen Applikationsart ersetzt es nicht das Debridement. Alleinige Antibiotikabehandlung ohne chirurgische Konsequenz ist nur im Ausnahmefall, z.B. moribunder Patient, zu rechtfertigen. Initiale Antibiose ohne umgehende Revision kann zudem zur Verschleierung der Symptome und zur chronischen Form der Osteitis führen.

\section{Fazit}

Im septischen Krankengut einer Unfallklinik ist das Problem völlig anders gelagert. Dort erhält man durch die Zuweisungen eine Negativselektion und zahlenmäßige Häufung von Patienten. Diese sind zumeist mehrfach voroperiert und kommen bereits mit funktionellen Defiziten zur Aufnahme. Zudem sind die Ursachen der Infektionen mannigfaltig. Entscheidend ist die Frage, was bei der kontroversen Diskussion in der Literatur als gesichert anzusehen ist!

- Der Zeitpunkt der Diagnosestellung und umgehend eingeleiteter aggressiver Therapie ist entscheidend für das Outcome des Patienten.

- Sorgfältige postoperative Kontrolle, klinisch und laborchemisch, ist daher unverzichtbar. Der akute Infekt ist ein Notfall, der umgehend revidiert werden muss. Im Akutfall darf durch mikrobiologische Testungen keine Verzögerung entstehen.

- Alleinige Antibiotikagabe ohne operative Revision ergibt keinen Sinn.

Ein wirklich sicheres und auch noch finanzierbares Verfahren gibt es nicht. Bei der ersten Infektrevision kann man u. E. nach bei nicht zu ausgeprägter Infektsituation das Implantat belassen. Nägel entfernen wir mittlerweile grundsätzlich, wenn es sich nicht nur um einen Subkutaninfekt an der Einschlagstelle handelt. Beim Infektrezidiv wird jedes Implantat entfernt.

Die objektiv geringen Infektraten erlauben es den behandelnden Ärzten nicht, ausreichende Erfahrung mit dieser schwerwiegenden Komplikation zu erzielen, um dann ohne Zeitverlust das im jeweiligen Einzelfall optimierte Behandlungsverfahren zu wählen und umzusetzen. Die Fragestellung des Patienten „Wie viel Erfahrung haben Sie eigentlich mit meiner Komplikation?" wird berechtigter [2]

\section{Literatur}

${ }^{1}$ Heppert V, Martens A, Wentzensen A. Hygiene im OP. OP Journal 2005; 21: 68-74

${ }^{2}$ Heppert V. Gelenkinfektion. In: Standardverfahren in der operativen Orthopädie und Unfallchirurgie. Hrsg. Ewerbeck V, Wentzensen A, Holz F, Krämer KJ, Pfeil J, Sabo D. Thieme Stuttgart, 2. Auflage, 2003: 816-818

${ }^{3}$ Heppert V. Wundinfektion. In: Standardverfahren in der operativen Orthopädie und Unfallchirurgie. Hrsg. Ewerbeck V Wentzensen A, Holz F, Krämer KJ, Pfeil J, 
Sabo D. Thieme Stuttgart, 2. Auflage, 2003: 807-809

${ }^{4}$ Diefenbeck M, Hofmann G. Behandlungsstrategie beim Frühinfekt nach operativer Frakturversorgung. Trauma Berufskrankh 2003; 5 (Suppl 2): 309-312

${ }^{5}$ Peters KM, Klosterhalfen B, Koberg K, Haubeck HD. Kosteneffiziente Labordiagnostik bei Knocheninfektionen . In: Jerosch (Hrsg) Infektionen des Bewegungsapparates. Thieme Stuttgart 1995: 48-54

${ }^{6}$ Hofmann G, Hierholzer C, Kirschner M, Mückley T. Einführung zur 17. Murnauer Unfalltagung ? Infektionen in der Traumatologie. Trauma Berufskrankh 2003; 5 (Suppl2): 290-292

${ }^{7}$ Heppert V, Glatzel U, Wentzensen A. Postoperative und bakterielle Osteitis - Neue Therapieansätze. Der Orthopäde 2004; 33: 316-326

${ }^{8}$ Tsukayama DT, Gustilo RB. Antibiotic Management of open fractures. In: Greene WB (Hrsg) Instructional Course lectures Vol. 39. American Academy of Orthopaedic Surgeons, Park Ridge, IL 1990: 487-490
${ }^{9}$ Cierny G, Mader JT. Approach to adult osteomyelitis. Orthop Rev 1987; 16: 95-106

${ }^{10}$ Costerton JW, Stewart PS, Greenberg EP. Bacterial Biofilms: A common cause of persistent infections. Science 1999; 284: $1318-$ 1322

${ }^{11}$ Stewart PS. Biofilm accumulation model that predicts antibiotic resistance of Pseudomonas aeruginosa biofilms. Antimicrob Agents Chemother 1994; 38: 1052 - 1058

${ }^{12}$ Bühler M, Kirschner S. Trauma Berufskrankh 2005; 7 (Suppl1): 105-109

${ }^{13}$ Arens S, Schlegel U, Printzen G, Ziegler WJ, Perren SM, Hansis M. Influence of materials for fixation implants on local infection. Experimental study of steel versus titanium DCP in rabbits. J Bone Joint Surg Br 1996 Jul; 78 (4): 647-651

${ }^{14}$ Hofmann G, Bär T, Bühren V. Osteosyntheseimplantat und früher postoperativer Infekt: Sanierung mit oder ohne Materialentfernung? Chirurg 1997; 68: 1175-1180
Dr. med. Volkmar Heppert

Leiter der Sektion:

Posttraumatische Osteitis

Prof. Dr. med. Andreas Wentzensen

Ärztlicher Direktor

BG-Unfallklinik Ludwigshafen

Ludwig-Guttmann-Str. 13

D-67071 Ludwigshafen 